Editorial

\title{
Al in Medical Imaging: Current and Future Status-Artificial Intelligence or Augmented Imaging?
}

\author{
Anirudh Kohli ${ }^{1}$ \\ ${ }^{1}$ HOD, Department of Imaging, Breach Candy Hospital, Mumbai,
} India,

Indian J Radiol Imaging 2021;31:525-526.

The next biggest disruption in medical imaging after the invention of computed tomography scan appears to be artificial intelligence (AI) in medical imaging. AI essentially refers to the ability of a computer to do tasks that are usually done by humans using their natural intelligence, such as in problem-solving and decision-making. Popular AI tools used in daily life are web search engines (Google), understanding human speech (Siri, Alexa, Augnito), self-driving cars (Tesla), or decision-making in strategic games such as chess.

Over the last few years, $\mathrm{AI}$ in medical imaging has been the most prominent buzz word. Medical literature has been flooded by articles on the value of AI in medical imaging. AI in medical imaging uses computerized algorithms to dissect imaging data, with the primary goal of detection of abnormalities and quantification of disease processes.

The general perception among medical imaging specialists is: AI is a tool that will replace them. The computer will analyze the images, detect the abnormalities, characterize it, and provide the diagnosis or differential diagnosis thus rendering medical imaging specialist redundant. Is this the real reality? What is the status currently or in the next $5 / 10$ years?

To date, computer-aided detection (CAD) has shown excellent sensitivity and specificity in specific disease processes such as breast cancer detection (microcalcification), ${ }^{1}$ colorectal cancer (polyps), and pneumonia detection. However, at no stage have they significantly exceeded human performance. The main drawback of CAD is that its goal is lesion detection; however, it does not provide any information on the biological aggressiveness of the lesion. Clinicians require medical imaging not only to detect lesions but also to correlate with symptoms, to assist in the decision-making process for further investigations and type of treatment, as well as to prognosticate regarding morbidity and mortality. Further, merely an increased sensitivity to lesion detection will result in an increasing number of clinically indolent insignificant lesions as well as false positives resulting in over diagnosis and unnecessary further procedures to determine the value of these lesions. Thus, any statistical demonstration of the endpoint of AI in medical imaging should be radiological/pathological endpoints that are clinically meaningful.

The present pandemic of SARS-CoV-2-severe acute respiratory syndrome corona virus-2-causing COVID-19-coronavirus disease-2019-pneumonia was a perfect case to examine the value of AI. It was actually a simple case of AI in a single disease with a fairly specific appearance. In detection, it may have matched human natural intelligence, but when it came to decision-making, CO-RADS-COVID-19 Reporting and Data System-scoring, or differential diagnosis, it was far inferior. ${ }^{2}$

Unless AI algorithms are trained to distinguish between benign abnormalities and clinically meaningful lesions, there will be no replacement for natural intelligence of medical imaging specialists. So, is AI of no value for the medical imaging? In fact, it is of immense value. There is a huge component of support in the form of assisted/augmented imaging.

Medical imaging has evolved from projection images to cross-sectional tomographic images. As technology evolves with the additional desire to image in more detail, this results in a large number of images and data. This can be quite daunting and time consuming for the radiologist. Computer-aided reconstruction of these cross-sectional tomographic images into projection images with exquisite detail as well as other reconstruction techniques such as multiplanar reconstructions, including curved multiplanar reconstructions, maximum intensity projections, minimum intensity projections, and volume rendering techniques have become standard parts of imaging to assist radiologists. There are numerous AI-based techniques that may further assist radiologists in improving workflow such as automated
Address for correspondence Anirudh Kohli, MD, DNB, DMRD HOD, Department of Imaging, Breach Candy Hospital, Mumbai 400026, India

(e-mail: dranirudhkohli@gmail.com).
DOI https://doi.org/ 10.1055/s-0041-1740168. ISSN 0971-3026. (c) 2021. Indian Radiological Association. All rights reserved. This is an open access article published by Thieme under the terms of the Creative Commons Attribution-NonDerivative-NonCommercial-License, permitting copying and reproduction so long as the original work is given appropriate credit. Contents may not be used for commercial purposes, or adapted, remixed, transformed or built upon. (https://creativecommons.org/ licenses/by-nc-nd/4.0/)

Thieme Medical and Scientific Publishers Pvt. Ltd., A-12, 2nd Floor, Sector 2, Noida-201301 UP, India 
protocols, smart cameras to assist in patient positioning and assessing contrast extravasation, triage studies for prioritization of reporting such as stroke, smart reporting, that is, linking text, images, and annotation, as well as automated communication with referring doctors. Assistance by AI is also found in time-consuming tasks such as vertebral body leveling, rib fracture detection, and assessment of bone age. AI can also provide assistance in increasing accuracy by screening for common cancers as well as in quantification, such as assessing the size of pneumothorax, and in providing functional information in aortic stenosis, fractional flow reserve, and magnetic resonance elastography. Essentially, if tedious repetitive tasks can be taken by the computer, this can improve workflow and hence time will be saved for the radiologists, so they may concentrate on the images.

Radiology practice is more than simple interpretation of the images; the need of the hour is for radiologist to incorporate clinical and laboratory findings into the interpretation of images to narrow down the differential diagnosis as well as guide the treating practitioner in decision-making regarding further investigations, therapy, or prognosis. A radiologist who ignores clinical and laboratory information would be reduced to an image interpretation specialist. The value of imaging findings in the clinical context will then be left to other physicians, a potentially dangerous situation as nonradiology experts in medical imaging may ask for unnecessary investigations to chase up innocuous and false positive lesions.

$\mathrm{AI}$ is not a threat to radiologist but a tremendous opportunity to assist radiologists in quickening the backend processes, improving workflow, increasing accuracy, and quantification of findings; so radiologist may use their time well to concentrate on image interpretation as well as not ignore clinical and laboratory information to provide a holistic picture to the treating clinician. If radiologists adhere to interpreting images in the context of clinical and laboratory findings (clinical radiologist), it is unlikely that AI will replace natural intelligence of the radiologists.

\section{References}

1 Artificial intelligence for breast cancer detection in mammography and digital breast tomosynthesis: State of the art: IoannisSechopoulos,JonasTeuwen,RitseMannad semcancer.2020.06.002

2 Artificial Intelligence of COVID-19 Imaging: A Hammer in Search of a Nail Ronald M. Summers Dec 22 2020https://doi.org/ 10.1148/radiol.2020204226 